\title{
Una Secuencia Didáctica para Abordar el Concepto de Calor en la Enseñanza de Estudiantes Preuniversitarios
}

\author{
José E. Simões-Neto(1), João R. R. T. da Silva(2), Maria E. B. Cruz ${ }^{(3)}$, Edenia M. R. do Amaral(1) \\ (1) Departamento de Química, Univ. Federal Rural de Pernambuco, Rua Dom Manoel de Medeiros, s/n, \\ Dois Irmãos-Recife, Pernambuco, Brasil (e-mail: euzebiosimoes@gmail.com ; edsamaral@uol.com.br) \\ (2) Unidade Acadêmica de Serra Talhada - Universidade Federal Rural de Pernambuco, Fazenda Saco, \\ s/n, caixa postal 063 - Serra Talhada, Pernambuco, Brasil. (e-mail: joaoratistenorio@gmail.com) \\ (3) Programa de Pós-Graduação em Ensino das Ciências - Univ. Federal Rural de Pernambuco, \\ Pernambuco, Rua Dom Manoel de Medeiros, s/n, Dois Irmãos-Recife, Pernambuco, Brasil. (e-mail: \\ mariaeduarda.b.cruz@gmail.com)
}

Recibido Jul. 9, 2014; Aceptado Sep. 23, 2014; Versión final recibida Nov. 30, 2014

\section{Resumen}

En este artículo se presenta y analiza una secuencia didáctica para abordar el concepto de "calor". El estudio se basa en la teoría del perfil conceptual de Mortimer, aplicada a un curso de estudiantes preuniversitarios, de la enseñanza media en Brasil. La comprensión de conceptos como "calor" que se discute en este trabajo es muchas veces determinante del éxito de los estudiantes en sus cursos de universidad. Se diseña y aplica una secuencia didáctica que busca relacionar el mundo material de los estudiantes con conceptos científicos sobre el calor. La propuesta incluye el uso de videos comerciales, software académico y un experimento virtual muy divulgado en sitios de la Internet. Los resultados muestran que la secuencia didáctica propuesta es adecuada para la mejor comprensión del concepto de calor por parte de los estudiantes.

\section{A Teaching Sequence for Addressing the Concept of Heat in the Teaching of Pre-University Students}

\begin{abstract}
In this paper a teaching sequence to describe and teach the concept of "heat" is presented and analyzed. The study is based on the theory of conceptual profile given by Mortimer, applied to a pre-university course from a high school, in Brazil. The comprehension of concept such as heat, discussed in this work is many times crucial for the success of student during their first courses in the university. A didactic sequence is developed and applied to relate the material world of students with scientific concepts such as heat. The proposal includes the use of commercial videos, academic software and a virtual experiment popular on the Internet. The results show that the proposed didactic sequence is appropriate for the better comprehension of the concept of heat by students.
\end{abstract}

Keywords: heat, teaching sequence, learning, scientific concepts 


\section{INTRODUCCIÓN}

Durante la década de los 70 se presentaron varias investigaciones apoyadas sobre la idea de considerar las concepciones previas de los estudiantes en el proceso de enseñanza-aprendizaje, tratando de modificarlas hacia la adquisición de conocimiento científico - entre otras propuestas, una se conoció como modelo de enseñanza por cambio conceptual y durante años fue sinónimo de aprendizaje de la ciencia, como afirman Niedderer, Goldberg y Duit (1991). En este contexto, surge la propuesta de que hay una variedad de formas de pensar acerca de un concepto que están asociadas a diversos factores y que no deben ser necesariamente abandonadas durante el aprendizaje de la visión científica del mismo. Apoyándose en esta premisa y tomándola como base epistemológica Bachelard, Mortimer (1995; 1996; 2000) proponen la noción del perfil conceptual.

El perfil conceptual se propone como un "sistema supra-individual de formas de pensamiento que pueden asignarse a cualquier individuo dentro de la misma cultura" (Mortimer, 2000, p. 80). Para el autor, el mismo concepto puede introducir diversos tipos de pensamientos filosóficos y de aspectos ontológicos que cuando son usados en los contextos apropiados, constituyen las zonas del perfil conceptual, y representan diferentes maneras de pensar y hablar acerca de la realidad, traduciendo la pluralidad del pensamiento conceptual (Amaral, Mortimer, 2004; Silva, Amaral, 2013; Mortimer, El-Hani, 2014).

Para este trabajo, utilizamos el concepto de calor propuesto por Amaral y Mortimer (2001), para el cual fueron identificadas y propuestas cinco zonas, a saber: realista, animista, sustancialista, empírica y racionalista. La primera zona, realista, aborda el calor a partir de las sensaciones térmicas, frío y caliente. La zona animista se refiere al calor como algo que da la vida, con una distinción ontológica respecto a la zona realista. En la zona de sustancialista, el calor es considerado como una sustancia, y encuentra su respaldo en los momentos históricos en los que se propuso la existencia del "calórico", una sustancia o elemento químico incluido por Lavoisier en sus tablas de clasificación de las sustancias conocidas. En la zona empírica, se considera la relación del calor con_el uso de instrumentos de medida para la cuantificación del mismo o de magnitudes asociadas, como el calorímetro, y principalmente, el termómetro. Por último, la zona racionalista, en la cual se define el calor a partir de una noción racional, una expresión matemática formal, que es lo que está más cerca de la visión científica del calor.

Uno de los retos para el programa de investigación sobre los perfiles conceptuales es la de su inserción concreta y su contribución al proceso de enseñanza y aprendizaje en el aula. Por lo tanto, en este trabajo hemos lanzado una propuesta para utilizar secuencias didácticas (secuencias de enseñanza y aprendizaje, TLS en inglés) preparadas a partir de las áreas de perfil conceptual. Las secuencias didácticas sirven para planificar los contenidos de enseñanza, maximizando el potencial de uso de diferentes metodologías, en un sistema interconectado de acciones en pos de aprendizaje (Nurkka, 2008; Firme, Amaral y Barbosa, 2009; Couso, 2011; Sarmento et al., 2013).

En esta perspectiva, podemos destacar el trabajo de Méheut (2005), que aborda la propuesta TLS en la investigación y apunta hacia aspectos importantes acerca de la elaboración de secuencias didácticas para la enseñanza de la ciencia. La autora afirma que la proposición y la aplicación de estas secuencias pueden ser analizadas mediante un modelo de cuatro componentes: profesores, estudiantes, mundo material y conocimientos científicos. Estos componentes se alinean en dos dimensiones: pedagógica y epistemológica, de acuerdo a la representación gráfica que se muestra en la figura 01 :

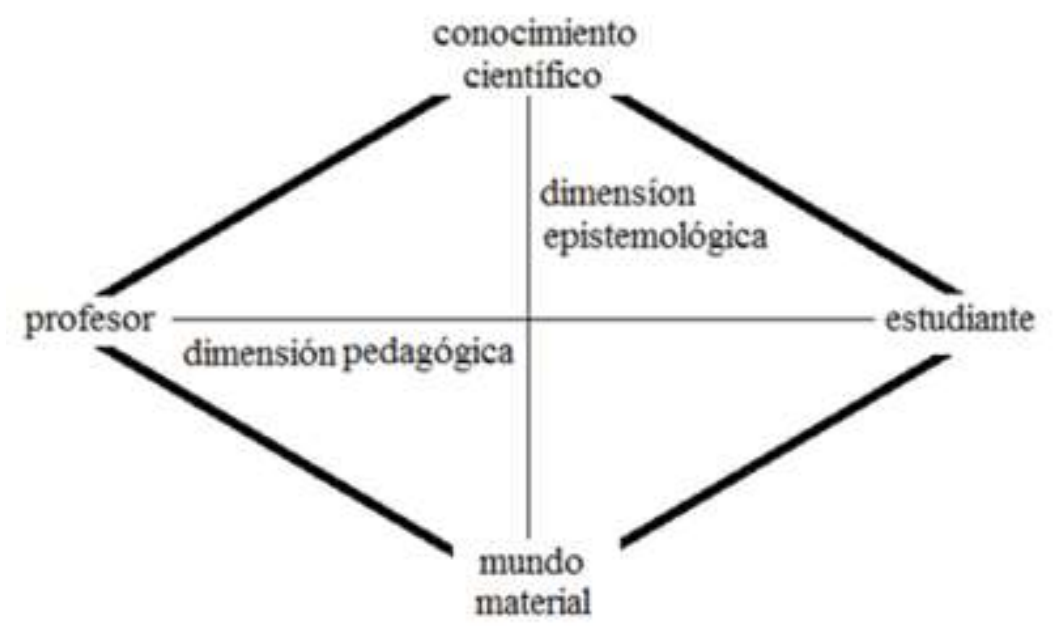

Fig. 1: Diamante de Méheut (2005), señalando las dimensiones de análisis en una secuencia didáctica 
Podemos entender las dimensiones epistemológicas y pedagógicas a partir de las relaciones entre los cuatro componentes mencionados. La dimensión epistemológica representa la construcción del conocimiento científico y todos los procesos relacionados con la interpretación del mundo material, y la dimensión pedagógica representa las interacciones en el aula entre profesor-estudiante y estudianteestudiante. Pensando en el diseño de las actividades de un TLS, y atendiendo a las dimensiones del diamante se hicieron propuestas que implicasen la integración entre los cuatro elementos que intervienen en la enseñanza y aprendizaje: el conocimiento científico, conocimiento del mundo real, el estudiante y el profesor. A la vista de lo expuesto, el objetivo de este trabajo es analizar las interacciones en el discurso a partir de una propuesta didáctica, tratando de identificar la aparición de zonas de concepto del calor en el aula y los indicios de aparición de la consciencia de estas zonas en los estudiantes. Las secuencias didácticas tienen una dimensión de investigación y otra de formación docente, y se caracterizan por ser una herramienta de investigación y enseñanza de una manera unificada.

\section{METODOLOGÍA}

Este trabajo fue desarrollado en un programa federal brasileño para la formación de maestros, el PIBID (Programa Institucional de Bolsas de iniciación a la docencia). Por lo tanto, es un trabajo centrado en la formación universitaria para la formación de maestros de educación básica. Dividimos la metodología en tres pasos: i) planificación y diseño de secuencia didáctica; ii) aplicación de la secuencia de intervención didáctica y recopilación de datos; iii) análisis de los datos y discusión de los resultados, evaluando la capacidad de la propuesta presentada para promover la aparición y consciencia de las zonas del perfil conceptual del calor.

\section{Planificación y diseño de secuencia didáctica}

Elaboramos la secuencia contemplando actividades que busquen relacionar el mundo material de los estudiantes con conceptos científicos como hace Méheut (2005), como una forma de incentivar la emergencia y la posible toma de consciencia de las zonas en el perfil del calor. Con este fin, seleccionamos algunos instrumentos didácticos que serían adecuados al debate propuesto en cada punto de la secuencia. Realizamos una búsqueda de videos comerciales que presentasen uno o más modos de pensar y hablar sobre el concepto de calor, según las cinco áreas del concepto.

Además, seleccionamos dos soportes informáticos para la discusión sobre la visión microscópica del concepto de calor y elaboramos un experimento para la presentación del formalismo matemático de este concepto. Al final, los materiales seleccionados y aplicados fueron episodios de las series: Los Caballeros del Zodiaco (Saint Seiya, Toei Animation), Smallville (Warner Bros.), El Pájaro Loco (Woody Woodpecker, Universal Pictures), Avatar: La Leyenda de Aang (Avatar: The Last Airbender, Nickelodeon) y Futurama (Fox Animation); y los programas computacionales La Transmisión del Calor (desarrollado por NOA-UFPB, Núcleo de Construcción de Objetos de Aprendizaje de la Universidad Federal da Paraíba, Brasil) y Fuente Térmica y cambio de Estado Físico (desarrollado por el Instituto Galileo Galilei); y el experimento de quema de cacahuetes en el Calorímetro, muy divulgado en sitios de internet.

\section{Aplicación de la secuencia de intervención didáctica y recopilación de datos}

Aplicamos la siguiente didáctica a 21 alumnos de una clase de tercer año de secundaria, el último año de estudio de educación básica en Brasil, en una escuela pública en el estado de Pernambuco, nordeste del país, ubicada en la ciudad de Serra Talhada (PE). La intervención fue realizada por dos investigadores, profesores de educación superior con experiencia previa en educación básica y con cinco intervenciones, descritas en la tabla 1.

La segunda, tercera y cuarta visita fueron clases de 50 minutos; y en la primera y última visita, trabajamos con clases hermanadas con un total de 100 minutos por visita, haciendo un total de siete clases para la aplicación de la secuencia completa. En Brasil, algunas clases son en par secuencial, llamadas clases gemelas. El punto de partida y el final de la secuencia didáctica fueron mayores (clases gemelas) debido a la necesidad de un debate entre los estudiantes e investigadores, así como para la presentación de la propuesta y culminación.

\section{Análisis de los datos y discusión de los resultados}

Se registra la secuencia didáctica en video, obteniendo los datos de la transcripción del mismo. Se realiza un análisis de las interacciones discursivas de los estudiantes, fijándonos cuando las líneas discursivas están relacionadas con el uso de las áreas del perfil de calor, y tratando de determinar si eran conscientes 
de la existencia de tal zona, o incluso si estaban construyendo concepciones científicas sobre el concepto. Para analizar el potencial de la secuencia, describimos los momentos de intervención didáctica siguiendo la aplicación diseñada, buscando resaltar los aspectos de motivación y el interés en las actividades propuestas, así como las evidencias de la construcción de nuevos significados para el concepto de calor. Para analizar la toma de consciencia en las zonas del concepto de calor, observamos al final de la secuencia, en la cual se presentaron videos comerciales que presentan modos de pensar y hablar sobre el concepto de calor, en diferentes contextos.

Tabla 1: Descripción de las actividades y objetivos de las intervenciones en la secuencia propuesta

\begin{tabular}{|c|c|c|}
\hline Momento & Actividad & Meta \\
\hline 1 & $\begin{array}{l}\text { Discusión y resolución de una situación- } \\
\text { problema. }\end{array}$ & $\begin{array}{l}\text { Obtener ideas sobre la sensación térmica (frío y } \\
\text { calor)-en relación con la zona realista del perfil } \\
\text { conceptual del calor. }\end{array}$ \\
\hline 2 & $\begin{array}{l}\text { Conferencia-la historia del concepto de } \\
\text { calor. }\end{array}$ & $\begin{array}{l}\text { Observar la génesis y el desarrollo del concepto en } \\
\text { comparación con los puntos de vista actuales, } \\
\text { incluidos los del sentido común. }\end{array}$ \\
\hline 3 & Actividad experimental & $\begin{array}{l}\text { Discutir el formalismo matemático mediante el } \\
\text { cálculo de la cantidad de energía (calor) emitido en la } \\
\text { quema de un grano de maní. }\end{array}$ \\
\hline 4 & Programas informáticos & $\begin{array}{l}\text { Discutir la visión microscópica de las simulaciones de } \\
\text { los procesos de transferencia de energía }\end{array}$ \\
\hline 5 & $\begin{array}{l}\text { Debate general con el uso de videos } \\
\text { (Recortes de episodios seleccionados de } \\
\text { Los Caballeros del Zodiaco, Smallville, El } \\
\text { pájaro loco, Avatar: La leyenda de Aang } \\
\text { y Futurama) }\end{array}$ & $\begin{array}{l}\text { Identificar las diferentes visiones del calor en la } \\
\text { secuencia presentada en clase. }\end{array}$ \\
\hline
\end{tabular}

\section{RESULTADOS}

Los resultados se presentan en dos sub-secciones: i) potencial de la secuencia didáctica; y ii) indicio de toma de consciencia de la multiplicidad de zonas del concepto de calor.

\section{Potencial de la secuencia didáctica}

Durante toda la intervención didáctica, los estudiantes mostraron interés y un alto grado de participación en las actividades. En el primer momento (tabla 1), se introduce la zona realista del concepto de calor mediante una situación-problema, que trataba la diferencia de sensación térmica en el contacto entre una persona y diversos materiales expuestos al sol, tales como plástico y metal. Podemos destacar los aspectos positivos relacionados con los trabajos en grupo, propuestos para la resolución del problema presentado. De los cinco grupos (de seis miembros cada uno), tres han presentado respuestas consideradas satisfactorias (que consiguen explicar la diferencia en la sensación al tacto de diversos materiales) y un grupo presentó una respuesta parcialmente satisfactoria (que ven la diferencia, pero sin dar explicaciones sobre el hecho). Los otros dos grupos dieron respuestas insatisfactorias. La receptividad a la segunda intervención fue menor, debido al alto grado de exposición y actividad teórica propuesta. Sin embargo, las preguntas sobre el contexto histórico en el desarrollo del concepto de calor estuvieron dentro del marco previsto, transitando entre el sentido común de los primeros conceptos, hasta las ideas científicas surgidas a partir del surgimiento de las ciencias, post-renacimiento.

Destacamos las actividades propuestas en el tercer y cuarto periodo (tabla 1). En estas, al utilizar el ordenador (trabajo con programas) 0 al interactuar con materiales concretos durante la actividad experimental en el laboratorio, fue posible desarrollar los aspectos metodológicos y de procedimientos, al colocar a los estudiantes en el centro del proceso de aprendizaje, desde el punto de vista de la interacción con el objeto de enseñanza. Durante la última intervención, los estudiantes estuvieron muy concentrados en los videos, anotando y delimitando cada manera de pensar y hablar sobre el concepto de calor, en los diversos contextos presentados por los clips audiovisuales. La actividad propuesta demuestra éxito en el debate final, en la cual surgieron las ideas que se presentan a continuación. 


\section{Indicio de toma de consciencia de la multiplicidad de zonas del concepto de calor.}

Los resultados apuntan a un posible reconocimiento de estudiantes, teniendo en cuenta que, al final de la intervención lograron identificar algunas áreas del concepto de calor en los videos, lo que también puede ser un signo de la validación de la secuencia didáctica. Es más, observamos que construyeron la noción de que las diversas opiniones pueden coexistir en un mismo individuo, cada una aplicada en un contexto particular. Vamos a presentar extractos de las transcripciones de los vídeos, en la que los estudiantes muestran que han identificado los conceptos de cada zona del perfil, y se posicionan en cada uno de ellos. Los nombres que se usan son ficticios y los investigadores son llamados PESQ1 y PESQ2.

\section{Zona realista}

JOSÉ: Pájaro Loco (...)_el uso de una bolsa de agua caliente introduciendo agua caliente para engañar a la enfermera, el pájaro Loco, térmica mayor (sic)... temperatura elevada.

PESQ1: ¿Algún video más?

JOSÉ: También tienes... Futurama, si no me equivoco. En un momento cuando la sensación térmica... la temperatura ambiente derrite el helado de ella, creo que llega a la conclusión del porqué el helado se derritió (sic). Y ella realmente... (Inaudible)... que es debido al calor que genera sensaciones.

PESQ1: ¿y está de acuerdo con este punto de vista?

JOSÉ: ¿De Futurama?

PESQ1: Tanto de Futurama como del Pájaro Loco, que se refiere a las sensaciones. ¿Estás de acuerdo? ¿No estás de acuerdo? ¿Crees que está bien, mal?

JOSÉ: En parte.

PESQ1: ¿En parte?

JOSÉ: Yo creo que (inaudible) sensaciones térmicas, lo que llega al cuerpo... viene al cuerpo... del ambiente hacia el cuerpo. Así sentimos, de hecho, una relación de temperatura... de hecho, creo eso es la sensación térmica. Pasas a distinguir lo que es frío y lo que es caliente. Vi eso en el Pájaro Loco. Y en Futurama también lo podemos observar. No es sólo la sensación térmica lo que hace que su helado se descongele. La sensación térmica proporciona el despertar del cuestionamiento de ella sobre el por qué el helado se derrite.

Nos damos cuenta que José identifica en dos videos la idea de calor en relación a las sensaciones térmicas. En su discurso, observamos que apunta a la importancia del concepto de calor relacionado con las sensaciones térmicas, porque a través de ello "pasas a distinguir lo que es frío y lo que es caliente", confirmando su posición de acuerdo con esta visión, que está todavía lejos del pensamiento científico. Sin embargo, observamos que se atribuye importancia a este tipo de conocimiento, ya que estas ideas son ampliamente utilizadas en el sentido común, contexto en el cual adquieren significado.

Algunos estudiantes se mostraron en desacuerdo con lo visionado en el contexto de los videos, debido a que los fenómenos que se presentan tienen poca credibilidad, como que el helado se derrita tan rápido en Futurama y la elevación en la temperatura provocada por la bolsa, ya que estando debajo de la almohada, provocó un gran aumento de la temperatura en el termómetro del Pájaro Loco. Otros estudiantes han identificado también tal visión en Avatar y Caballeros del Zodiaco, aunque sin emitir una justificación razonada para esas visiones.

\section{Zona Animistas}

JOSÉ: En Futurama podemos observar que cuando el chaval intenta explicar (...) el calentamiento global... cuando ella coge el rayo de sol (sic), los dibujos animados lo hace muy bien, un chaval sale para el trabajo, por así decirlo, con su maletín, caminando a través del Espacio Sideral hasta encontrar la Tierra... y es destrozado por los gases de efecto invernadero. Entonces, proporciona una animación.

El estudiante José identifica la visión animista en Futurama, pero no está de acuerdo con la idea. Sin embargo, posteriormente, afirma estar de acuerdo parcialmente, porque considera que el calor es importante para el mantenimiento de la vida. Otros estudiantes se han posicionado en la misma forma:- 
JUCA: El calor... está (sic) conectado directamente a la vida, porque el calor es la energía que está siendo transferida (...) nosotros vamos a tener calor en las cosas. Es decir, está directamente conectado... está (sic) relacionado... es algo que va... junto con la vida.

En el discurso de Juca, nos damos cuenta de la importancia que le da a la visión animista. Por lo tanto, podemos considerar que admite esta forma de pensar, incluso siendo discrepante de la visión científica. Esto ratifica la idea del perfil conceptual, en el cual las ideas adquieren significado en contextos específicos desde el momento en el cual presentan valor práctico.

\section{Zona Sustancialista}

JOSÉ:... el rayo de calor, en ese caso, ¿no? Para evaporar... para cambiar el estado físico de una cantidad absurda de agua... pero eso no viene al caso.

PESQ2: Bueno... ¿Están de acuerdo con la visión de que el calor es una sustancia? En el contexto que ustedes entiendan, ¿OK? ¿Creen en esta visión del calor como una sustancia?

En el momento en el que el investigador 2 lanza la pregunta, nos dimos cuenta que la mayoría de los estudiantes no están de acuerdo con esa visión, no hay manifestaciones a favor. Tal vez los estudiantes se hayan quedado algo confusos, ya que la visión sustancialista es muy común en la escuela, desde su presentación en los primeros grados de educación básica.

\section{Zona Empírica}

José y Pedro respondieron que en el Pájaro Loco se percibe la visión empírica de calor. Entonces el estudiante Juca explicó que en caballeros del Zodiaco también se explicitaba la zona.

JUCA: En caballeros del zodiaco, el maestro... habla... que con el uso del termómetro medirá... digamos... el movimiento de las partículas... de los átomos.

PESQ2: él habla de la medida, pero no usa el instrumento. No aparece...

JUCA: Si... no usa el instrumento, pero da la explicación sobre lo que sería el uso del termómetro.

(Con discurso de Juca, Pedro interviene con algo que percibió en Avatar, mostrando que la visión empírica, se ha manifestado también sin la presencia de un instrumento, pero con alguna forma de medida de la temperatura)

PEDRO: Sin necesidad de utilizar un instrumento, también se ve en Avatar. Cuando se presentan. (Inaudible) en el globo... llegan cerca del volcán, la temperatura se iguala en cierta forma y el globo empieza a perder altura.

Para Pedro, el hecho de la pérdida de altura del globo induce la idea de que la temperatura se está equilibrando, sin la necesidad de usar un termómetro. Cuando se les pregunta, los estudiantes están de acuerdo que el punto de vista empírico es válido en algunos contextos. En este fragmento, el discurso de Pedro puede representar una comprensión de los intercambios de calor para alcanzar el equilibrio térmico, siendo la temperatura el indicador de este proceso. Sin embargo, no está claro si el estudiante hizo la distinción entre calor y temperatura.

\section{Zona racionalista}

CARLOS: Los caballeros del Zodiaco... porque, cuando está explicando, dice que... es sobre el movimiento de los átomos... que cualquier material... contienen átomos... solamente que están muy parados (sic). Es cuando está al cero absoluto.

En el discurso de Carlos aflora el entendimiento de que el calor es no sólo sensación y temperatura, sino que hay una relación con la constitución de la materia y el movimiento de las partículas. Aunque no se ha utilizado la noción racional para el cálculo de la cantidad de calor implicada en un proceso, los estudiantes expresan un entendimiento que va más allá de los aspectos sensibles o fenomenológicos, alcanzando un 
nivel explicativo de los fenómenos. Los estudiantes indicaron que están de acuerdo con la visión racional del concepto de calor.

\section{DISCUSIÓN}

Llevamos a cabo una discusión general sobre lo visionado en cada vídeo, explicitando las zonas que hemos identificado previamente, durante la planificación. Para averiguar si los estudiantes tenían la misma percepción de visiones de las zonas que nosotros preguntamos (identificadas durante la planificación), y obtenemos los resultados presentados en la tabla 2. En la Tabla $\mathrm{N}$ es el número de alumnos que están de acuerdo con nuestra percepción (Total 21 estudiantes)

Tabla 2: Número de estudiantes que aceptan las visiones

\begin{tabular}{|c|c|c|}
\hline Video & Zona & N \\
\hline $\begin{array}{c}\text { Los Caballeros del Zodiaco y } \\
\text { Futurama }\end{array}$ & Realista & 1 \\
\hline Futurama & Animista & 1 \\
\hline Smallville & Sustancialista & 13 \\
\hline El Pájaro Loco & Empírica & 11 \\
\hline $\begin{array}{l}\text { Los Caballeros del Zodiaco y } \\
\text { Avatar: La Leyenda de Aang }\end{array}$ & Racionalista & 10 \\
\hline
\end{tabular}

El número total de 21 estudiantes presentes en la última intervención no coincide con la suma de las cifras en la tabla 2, porque algunos estudiantes levantaron su mano más de una vez, demostrando que identifican las áreas en más de un vídeo, según lo propuesto por la teoría del perfil conceptual: En un mismo individuo pueden coexistir varias zonas.

Según la tabla 2 y los extractos analizados, vemos que la mayoría de los estudiantes conciben más de una forma de pensar sobre el concepto de calor, y aplican esos modos en contextos específicos. Esto nos puede dar pistas sobre una posible toma de consciencia, puesto que las ideas alternativas sobre el concepto de calor se utilizan intuitivamente en la vida cotidiana. Para adquirir la consciencia es necesario un ejercicio de metacognición, que se puede inducir mediante ciertos tipos de actividades, tal y como se sugiere en la propuesta didáctica. De esta forma, el alumno reflexiona sobre su forma de pensar y analiza sus propias ideas, tomando consciencia de las mismas usando la mediación y la supervisión del profesor.

En términos generales, la discusión con los estudiantes también nos mostró que cada visión de las zonas puede tener utilidad al ser incluida en determinados contextos, y no son consideradas necesariamente como erróneas. Para entender el significado que algunos conceptos ganan en contextos diferentes de la escuela 0 del contexto científico, los estudiantes pueden empezar a entender que la ciencia presenta una visión específica y particular del mundo y que son admisibles otras formas de pensar. En este proceso, pueden ser discutidos muchos aspectos de la enseñanza de las ciencias, como el papel de la ciencia en la vida contemporánea, la valoración de otras visiones del mundo, teniendo en cuenta que pueden presentar un poder explicativo limitado y las implicaciones socio-culturales de desarrollo científico y tecnológico. Este tipo de discusión puede conducir a los estudiantes a una consciencia de las zonas del perfil conceptual del calor y situar el aprendizaje de conceptos científicos en el escenario más amplio del pensamiento humano.

\section{CONCLUSIONES}

El análisis de los datos muestra que la secuencia brindó una construcción del conocimiento sobre el concepto de calor. En el discurso de los alumnos, en ciertos momentos, se percibe la construcción de las nociones científicas que se confrontan con otras visiones sobre el concepto de calor desde el análisis de situaciones concretas presentadas en diferentes recursos didácticos.

Por los resultados obtenidos de la observación de cada paso de la intervención en la secuencia didáctica, creemos que tiene capacidad para discutir el perfil conceptual del calor y que encamina la enseñanza y los procesos de aprendizaje de una forma satisfactoria.

Sobre la toma de consciencia, los estudiantes usan en otros contextos visiones del calor que difieren de la visión científica. Observamos que no consideran que la visión científica sea la única aceptable, y que algunas concepciones pueden tener sentido si se utilizan en los contextos adecuados. 
La dimensión de la formación de profesores es aspecto fundamental en las secuencias didácticas como una herramienta para la enseñanza y la investigación, lo que demuestra su importancia en la formación universitaria, especialmente de profesores de ciencias, que deben reconocer en su práctica las dimensiones pedagógicas y epistemológicas, las cuales son fundamentales para el diseño de una buena secuencia didáctica. También, el reconocimiento de la multiplicidad de modos de pensar y hablar sobre el concepto de calor para estudiantes pre-universitarios puede tener aspectos positivos, en especial para aquellos que seguirán las carreras de ciencias exactas e ciencias de la ingeniería en su futura jornada universitaria.

\section{REFERENCIAS}

Amaral, E.M.R. y E.F. Mortimer. Uma proposta para o perfil conceitual para o conceito de calor, Revista Brasileira de Pesquisa em Educação em Ciências: 1(3), 1-16 (2001).

Amaral, E. M. R. y E. F. Mortimer. Un perfil conceptual para entropía y espontaneidad: una caracterización de las formas de pensar y hablar en el aula de Química. Educación Química, v. 15, n. 03, p. 01-75, (2004).

Couso, D. Las Secuencias didácticas en la enseñanza y el aprendizaje de las ciencias: modelos para su diseño y validación, in Caamaño, A. Didáctica de la física y la química. Graó, Barcelona, Espanha (2011).

Firme, R.N., E.M.R. Amaral y R.M.N. Barbosa. Análise de uma sequência didática sobre pilhas e baterias, anais do XIV Encontro Nacional de Ensino de Química, Curitiba, Brasil, 21 a 24 de julio (2008).

Niedderer, H., F. Goldberg, R. Duit. Towards Learning Process Studies: A review of the Workshop on Research in Physics Learning, in R. Duit, F. Goldberg, H. Niedderer. Research in Physics Learning: Theoretical Issues and Empirical Studies. IPN, Kiel (1991).

Méheut, M. Teaching-learning sequences tools for learning and/or research. Springer, Paris, França (2005).

Mortimer. E. F. Conceptual Change or Conceptual Profile Change? Science \& Education. Netherlands: Kluwer Academic Publishers. p. 268-283 (1995).

Mortimer, E.F. Construtivismo, mudança conceitual e ensino de ciências: pra onde vamos? Investigações em Ensino de Ciências: 1(1), 20-39 (1996).

Mortimer, E.F. Linguagem e Formação de Conceitos no Ensino de Ciências. Editora UFMG, Belo Horizonte, Brasil (2000).

Mortimer, E.F. y C.N. El-Hani. Conceptual Profiles: A Theory of teaching and learning scientific concepts. Springer, New York, USA (2014).

Nurkka, N. Use of Transfer Teaches in Developing a Teaching-Learning Sequence: a case study in physioterapy education in Filand. NorDiNa. 4 (1), 201-214 (2008).

Sarmento, A. C. H., R. R. Muniz, N. R. Silva, V. A. Pereira, M. A. S. Santana, T. S. Sá y C. N. El-Hani. Investigando Princípios de Design de uma Sequência Didática sobre Metabolismo Energético. Ciência e Educação. 19 (3), 573-598 (2013).

Silva, J.R.R.T. y E. M. R. Amaral. Proposta de um Perfil Conceitual para Substância. Revista Brasileira de Pesquisa em Educação em Ciências. 13 (3), 53-72 (2013). 